\title{
A unified science of the non-conscious mind?
}

\section{Beatrice de Gelder and Marco Tamietto}

The notion of non-conscious processing as used in cognitive and affective neurosciences has developed largely independently from the notion of the unconscious that is familiar from Freudian psychoanalytic tradition, as mentioned in our recent Review (Neural bases of the non-conscious perception of emotional signals. Nature Rev. Neurosci. 11, 697-709 (2010)) ${ }^{1}$. The neuroscientific notion of non-conscious processing is largely defined by the experimental procedures that are currently available to neuroscientists to interfere with (or block) awareness of stimuli presented in carefully controlled laboratory settings. Nevertheless, since its beginning, the scientific study of the mind/brain - regardless of whether one views it as starting with Darwin, Wundt or Freud - has postulated the existence of mental processes that are not accessible to consciousness. Is the time ripe for striving towards a unified theory of unconsciousness? Behind terminological differences lies the more fundamental question raised by O'Brien in his correspondence; of whether, and to what extent, apparently similar notions and phenomena in neuroscience and psychoanalysis are related (Unconscious by any other name... Nature Rev. Neurosci. 7 Apr 2011 (doi: 10.1038/nrn2889-c1)) 2 .

Scientific constructs are defined by their theoretical and methodological context. Recasting the Freudian notion of unconscious in the context of modern neuroscience also requires a translation of the methodological and theoretical perspective. This may explain why accounts of Freudian concepts in a neuroscientific perspective have usually proved difficult. A fruitful interdisciplinary dialogue can be helped by comparing the constructs of 'non-conscious' and 'unconscious' emotions along several dimensions that seem relevant to both affective neuroscience and psychoanalysis.

A first dimension is the distinction between process and content. Neuroscience and psychoanalysis grant that most of the processes carried out by the mind/brain occur outside awareness but substantially contribute to it (for example, we are not aware of neural spikes although these are at the roots of conscious vision and memory). Along these lines, there have been interesting efforts to recast Freudian concepts in neuroscientific terms $s^{3}$. For example, the Freudian distinction between the primary (unconscious) and secondary (conscious) process has been linked to evidence that the brain has different modes of functioning and to a hierarchical organization of neural systems ${ }^{4}$.

With respect to contents, affective neuroscience and psychoanalysis both deal with the affective determinants of behaviour. In cases in which these determinants are represented by external events, both disciplines accept that there is a substantial non-conscious component. In neuroscience it may be helpful to distinguish between behavioural determinants that operate completely outside awareness and cannot possibly gain access to awareness (such as some odours or pheromones), and other determinants (such as visual or sensory events) that normally have access to awareness. In the latter case, when external emotional events fail to reach the level of consciousness it is because fewer neural resources dedicated to their processing are available. This may be due to neural damage that blocks processing in certain brain areas, or may reflect the fact that processing resources are allocated elsewhere, such as in the case of attentional unawareness. In psychoanalysis, when an event that is potentially accessible to consciousness fails to do so, this is generally attributed to a surplus of processing, such as when an active mechanism of suppression has prevented the event from reaching awareness. Therefore, the two disciplines seem to differently conceive the processes by which an emotional event can become a content of consciousness, and the reduction versus increase of processing resources can be another potentially important testing ground for comparing concepts across disciplines.

Another relevant dimension is the relation between awareness and phylogenetic and/ or ontogenetic development. Although it seems, at the clinical level, that psychoanalysis focuses on the unique individual experience, its goal is also to connect the individual experience with its phylogentic significance. It may be that neuroscientific approaches to non-conscious emotions have not yet gone far enough to envisage building bridges between ontogeny and phylogeny. For example, some interdisciplinary proposals suggest that our individual responses to emotional events are initially guided by non-conscious action schemata $^{5,6}$. These primary emotions are mapped during interactions in early infancy and influence other conscious cognitive processes such as attention and memory ${ }^{7}$. In affective neuroscience, however, there has been a tendency to assume that external events of which we are not aware are represented by the mind/ brain as having exactly the same identity and the same conceptual, logical and semantic properties as when we are aware of them. Granting the existence of a layered emotional system that has substantial similarities across species in its core components leads to the consideration that when an affective stimulus is processed non-consciously, it is processed as part of another - more primary - affective network than that embedded in the cortical conscious systems ${ }^{8}$. In fact, in that case, it is processed in connection with more basic determinants of behaviour.

A few brain-imaging studies have already shown that partly different brain networks respond to perception with or without visual awarenss ${ }^{9}$, leading to different behavioural and physiological responses ${ }^{10}$. Maybe we need to reckon much more with the possibility that affective events - whether perceived or remembered - are in one definition consciously perceived, and in another non-conciously perceived. This may be an important contribution of clinical science to basic affective neuroscience.

Beatrice de Gelder and Marco Tamietto are at the Cognitive and Affective Neuroscience Laboratory, Tilburg University, 5000 LE Tilburg, The Netherlands.

Beatrice de Gelder is also at the Athinoula A. Martinos Centre for Biomedical Imaging, Massachusetts General Hospital, Harvard Medical School, Charlestown, Massachusetts 02129, USA

Correspondencet to B.d.-G. e-mail: degelder@uvt.nl doi:10.1038/nrn2889-c2

Tamietto, M. \& de Gelder, B. Neural bases of the nonconscious perception of emotional signals. Nature Rev. Neurosci. 11, 697-709 (2010).

2. O'Brien, D. J. Unconscious by any other name... Nature Rev. Neurosci. 7 Apr 2011 (doi: 10.1038/ nrn2889-c1).

3. Schore, A. N. Relational trauma and the developing right brain: an interface of psychoanalytic self psychology and neuroscience. Ann. NY Acad. Sci. 1159, 189-203 (2009).

4. Carhart-Harris, R. L. \& Friston, K. J. The default-mode, ego-functions and free-energy: a neurobiological account of Freudian ideas. Brain 133, 1265-1283 (2010)

5. Bowlby, J. A secure base (Routledge, London, 1988). 6. Greenwald, A. G. New look 3. Unconscious cognition reclaimed. Am. Psychol. 47, 766-779 (1992). Ardito, R. B., Adenzato, M., Dell'Osbel, G., Izard, E. \& Veglia, F. Attachment representations in adults with congenital blindness: association with maternal interactive behaviors during childhood. Psychol. Rep. 95, 263-274 (2004).

8. Panksepp, J. Affective consciousness: core emotional feelings in animals and humans. Conscious Cogn. 14, 30-80 (2005).

9. Williams, L. M. et al. Mode of functional connectivity in amygdala pathways dissociates level of awareness for signals of fear. J. Neurosci. 26, 9264-9271 (2006)

10. Tamietto, M. et al. Unseen facial and bodily expressions trigger fast emotional reactions. Proc. Natl Acad. Sci. USA 106, 17661-17666 (2009). 